\title{
Mindfulness for Psychologists: Paying Kind Attention to the Perceptible
}

\author{
Paul Grossman
}

Published online: 26 May 2010

(C) Springer Science+Business Media, LLC 2010

\begin{abstract}
Basic definitions, assumptions, and working principles of the Buddhist and mindfulness-based intervention conception of "mindfulness" are characterized. Fundamental distinctions are drawn between Buddhist psychology and mainstream Western behavioral science in terms of aims, types of acquired knowledge, and professional training requirements. Particular emphasis is placed upon issues related to clinical intervention and biopsychosocial dysfunction. An argument is made and examples presented to show that mindfulness is best understood within an experience-based psychological perspective, integrating cognitive, affective, and ethical dimensions. The systematic nature of the development of mindfulness is illustrated by examination of an ancient program aimed at mindful awareness of breathing. Comparisons are made between developmental progression of mindful awareness and a neuroscience-based model of the ontogenesis of capacities of human consciousness; also, the act of awareness or being conscious is distinguished from the capacity for consciousness. In conclusion, acknowledgement of inherent differences - and tensions - between Buddhist and Western psychologies may actually serve to safeguard the concept of mindfulness from being denatured, banalized, or distorted. The potential of mindfulness-based approaches may, in turn, be enhanced for contributing to critical aspects of healthcare, quality of life, and wellbeing.
\end{abstract}

P. Grossman $(\bowtie)$

Division of Internal Medicine, Department of Psychosomatic

Medicine, University Hospital Basel,

Hebelstrasse 2,

CH-4031 Basel, Switzerland

e-mail: pgrossman@uhbs.ch
Keywords Mindfulness · Mindfulness-based stress reduction · Mindfulness-based interventions - Meditation . Psychotherapy $\cdot$ Respiration $\cdot$ Breathing $\cdot$ States of consciousness $\cdot$ Neuroscience $\cdot$ Phenomenology

\section{Introduction}

The history of academic psychology and psychotherapy in the twentieth century is heavily dominated by behaviorist concepts and theory. During this period, subjective perception and personal experience were generally depreciated as sources of psychological understanding, seen as outside the realm of verifiable scientific knowledge, and forgotten as the major aspect of individual human existence and awareness through which all cognitive and affective processes are ultimately filtered (Depraz et al. 2003). Therefore, it is more than ironic that mindfulness-a concept central to the inherently introspective-i.e., subjective and personal, psychology of Buddhism should have been ushered into mainstream Western psychological thinking and practice by behavioral therapists (Segal et al. 2002). In fact, in recent years, a surge of interest, primarily from cognitive-behavioral therapists, has begun to bring "mindfulness" into the vocabulary of mainstream psychology (see Clinical Psychology: Science and Clinical Practice, vol.10, 2003).

In this paper, I examine some of the most basic definitions, assumptions, and working principles of the Buddhist conception of mindfulness, as well as how these contrast with our mainstream Western behavioral science approaches toward the "self", biopsychosocial dysfunction, and clinical interventions. It is my hope that this stillpreliminary discussion of mindfulness may serve to plant the scientific research within a psychological context that, 
while unfamiliar, is thought-provoking and informative. Otherwise, the inherent tensions between Buddhist and Western psychologies, potentially so useful to consider, may go unnoticed and become lost and trivialized in the usual empirical analysis and positivist perspective.

\section{Definitions of Mindfulness}

Mindfulness is typically defined in terms of "awareness and attentiveness to immediate experience." The notion that we can be aware of and attend to our perceptible mental states and processes in the present moment may appear to fit well into modern psychological theories of attention and cognitive function. It may even seem absurdly self-evident and banal. However, hidden behind the simplicity of concepts and the commonality of terminology is an approach to the mind that is a radical departure from those of modern psychology, particularly behaviorism. Mindfulness from the Buddhist perspective encompasses and is at the same time embedded in a range of not only cognitive, but also emotional, social, and ethical dimensions, which extend far beyond the usual compartmentalization of conditioning, attention, and awareness of academic psychology. Just these differences are essential to an adequate understanding of Buddhist mindfulness and the role it plays in a psychological epistemology. Embracing a more circumscribed conception of mindfulness that easily fits, as just one more technique, into the armamentarium of behavioral and psychotherapeutic interventions neither does justice to the original idea nor represents the scientific investigations and literature on mindfulness-based interventions to this point.

It should be mentioned that almost all published mindfulness-based intervention studies employ a particular format, referred to as mindfulness-based stress reduction (MBSR; Kabat-Zinn 1990) or its very close cousin, mindfulness-based cognitive therapy (Segal et al. 2002). This approach, although only occasionally referring to its Buddhist roots, still largely adheres to the basic goals, principles, and procedures of traditional insight (Vipassana) meditation, of which mindfulness is a central component. The strategy I take in this paper is aimed at illustrating not only the promise of mindfulness for clinical intervention but also the richness of mindfulness both for the instructor and the instructed.

The concept of mindful awareness, or mindfulness, dealt with here specifically refers to that approach originating in earliest Buddhist treatises, but is neither religious nor esoteric in nature. "Mindfulness" is, of course, a common English word and can have a variety of meanings and usages. However, none fully or even approximately embodies the Buddhist notion of mindfulness, although there is sometimes an overlap. For example, Webster's
Dictionary (1998) defines mindfulness as the quality of "having in mind," being "aware, heedful or careful of something (to be mindful of the danger)." The internet Hyperdictionary (http://www.hyperdictionary.com/dictio nary/mindfulness) defines mindfulness as "the trait of staying aware of (paying close attention to) your responsibilities," whereas Roget's Thesaurus provides the following synonyms: care, carefulness, caution, gingerliness, heed, heedfulness or regard (1995).

Colloquial use of "mindfulness" often connotes being heedful or taking care within a clearly evaluative context: A parent tells a child, "mind your manners, or mind your language," implying to take care to behave in a culturally prescribed manner. "Mindful of the poor road conditions, he drove slowly." "What is man, that thou art mindful of him? (Psalms, viii. 4)." "I promise you to be mindful of your admonitions," or "always mindful of family responsibilities." All these formulations reflect an emphasis on carefully paying attention so as to not reap the consequences of heedless behaviors.

A contemporary and relatively popular scientific characterization of mindfulness refers to openness to novelty, alertness to distinction, sensitivity to variation of context, awareness of multiple perspectives, and orientation in the present (Langer 1997; Sternberg 2000). This primarily scientific application of the term implies a cognitive awareness and assessment of situational variability in the present circumstance, as well as a practical and goal-directed development of concrete skills, which facilitates differentiated perspectives. Any of the above classes of definitions overlap with Buddhist mindfulness only to the extent that they orient to aspects of awareness and/or the present moment.

Mindfulness, as derived from Buddhist treatises, is characterized by dispassionate, non-evaluative, and sustained moment-to-moment awareness of perceptible mental states and processes. This denotes continuous, immediate awareness of physical sensations, perceptions, affective states, thoughts, and imagery. Mindfulness is nondeliberative: It implies sustained paying attention to ongoing mental content in the present moment without thinking about, comparing, or in other ways evaluating the ongoing mental phenomena that arise during periods of practice. Because of this emphasis on direct awareness, minimally filtered by active evaluation or analysis, mindfulness is often described as "bare attention" to mental events and processes (Epstein 1995). Thus, mindfulness may be seen as a form of naturalistic observation, or more precisely participant-observation, in which the objects of observation are the perceptible mental phenomena that arise during all states of waking consciousness.

The definition, just offered, of mindfulness from a Buddhist perspective may still seem very amenable to 
Western psychological and cognitive-behavioral interpretation and integration. However, close scrutiny may reveal significant disparities with a purely cognitive approach: The development of "dispassionate, non-evaluative and sustained" awareness may sound simple and technical-a cool and neutral observation of one's own cognitive events and processes that are available for perception. However, as anyone who has attempted to develop this skill will corroborate, paying attention in this manner is difficult and complex. For one, there is the issue of maintaining sustained awareness as opposed to drifting off unawares into thoughts and images (typically away from the present moment and into the past or future). This tendency is not unrelated to the other defined characteristics of mindfulness, namely the "non-evaluative" and "dispassionate" dimensions. Both imply an approach largely unfamiliar to our normal waking thought process in which we tend to be extremely evaluative and often emotionally reactive, and which typically draws us away from sustained awareness. It is precisely at this juncture- between sustained moment-tomoment awareness and suspension of evaluation and emotional reactivity-where the affective and ethical dimensions enter into mindfulness.

To go into depth regarding affective and ethical factors in mindful awareness is beyond the scope here, but a few examples may suffice to suggest how sustained attention is facilitated and strengthened by simultaneous cultivation of ethical behavior and a particular affective mindset. Attention to one's sensations, cognitions, and emotions will ordinarily unleash cascades of thoughts, emotions, rumination, and evaluation for those unaccustomed to the practice of mindful awareness (often for those with experience, as well!). Therefore, it is necessary to nurture a certain affective state of mind in order to prevent such distraction from constantly occurring during mindfulness practice. Within Buddhist psychology, this includes the cultivation of an intention to develop kindness, patience, tolerance, gentleness, empathy, nonstriving acceptance and openness (a good description can be found in Shapiro and Schwartz 2000). The immediate focus of this intentional attitudinal mindset is the process of mindfulness itself-attempting, over and over, to accept and be kind to all the levels of unpleasant inner experiences that may arise from moment to moment (i.e., uncomfortable physical sensations, thoughts, or emotions), as well as the transitions (e.g., from a pleasant sense of calm to agitation, tiredness, or boredom) that may surface during mindfulness practice. As the mindfulness practice unfolds, the ability to maintain concentration and the acquisition of a kinder, more tolerant, and open mode of inquiry are thought to reinforce each other, providing a kind of synergy of process.

Analogously, ethical behavior is considered to be essential for the development of mindfulness because it frees the individual from the agitation that unethical behavior produces. Unethical behavior in Buddhism, by the way, solely includes those acts that do harm to self and others (physical harm, slandering, lying, stealing, sexual misconduct, etc.). In Buddhist psychology (and in most people's experience), unethical acts often create a sense of shame, guilt, and/or emotional repression that are likely to disquiet mental functioning. Such a restive state is seen as unconducive to the development of a calm and concentrative state that is a necessary precondition to fostering mindfulness (e.g., Goldstein 1993). As with the cultivation of more gentleness, kindness, generosity, and tolerance, Buddhist psychology maintains that not only does ethical behavior reinforce mindfulness, but mindfulness is seen to reinforce ethical behavior as well.

Accordingly, this conception of mindfulness is expansive and inherently transformative in nature and, as mentioned before, is at the foundation of MBSR. Any more limited concept of mindfulness has not been employed in the core scientific literature on mindfulness-based interventions (with the exception of the substantially different operationalization of Langer 1997). Therefore, there is no evidence to date that a more limited conception will serve as an effective behavioral intervention.

It is also important to make explicit certain assumptions underlying the Buddhist concepts and approach of mindfulness (Buchheld et al. 2001; Grossman et al 2004; Kabat-Zinn 1993; Nanamoli and Bodhi 1995): (1) Humans are ordinarily largely unaware of their moment-to-moment experience, often operating in an "automatic pilot"" mode. (2) The lack of awareness of own mental content and processes provides rich soil for misperceptions and self-delusion. (3) We are capable of developing the ability to sustain nonjudgmental, moment-to-moment, and highly discerning awareness of mental content. (4) Development of this ability is gradual, progressive, and requires regular practice. (5) Moment-tomoment awareness of experience will provide a richer and more vital sense of life, inasmuch as experience becomes more vivid and active mindful participation replaces unconscious reactiveness. (6) Such persistent, nonevaluative observation of mental content will gradually give rise to greater veridicality of perceptions. (7) Because more accurate perception of one's own mental responses to external and internal stimuli is achieved, additional information is gathered that will enhance effective action in the world and lead to a greater sense of control.

In light of these and other assumptions, it is important to keep in mind that the basic goals of psychotherapy and mindfulness practice are not identical. Psychotherapy is almost always directed toward elimination of presenting complaints. The primary aim of mindfulness practice is the development of insight and understanding of the mind in relation to all experience achieved by means of the 
cultivation of a moment-to-moment, nonjudgmental, but highly discerning awareness (Kabat-Zinn 2003).

\section{Toward an Understanding of the Development of Mindfulness}

\section{Discourse on Full Awareness of Breathing}

Insight meditation and mindfulness practice are based on an ancient method of inquiry. However, recently, this approach has been seen as a central method of acquiring phenomenological knowledge (Depraz et al. 2003; Varela et al. 1991). At the core is a systematic program of acquisition that was written down some 2,300 years ago. It may be instructive to briefly examine segments of one major text describing this approach and to elaborate upon the empirical underpinning of this method in terms of currentday science. Most mindfulness practices commence with the observation of breathing. The treatise, entitled the "Discourse on Full Awareness of Breathing," may be the oldest, detailed manual of a meditation procedure. An abbreviated version is presented below (adapted from (Nanamoli and Bodhi 1995):

"Now, how is mindfulness of in- and out-breathing developed and pursued so as to bring full awareness of the body, feelings, mental formations and the way things are in and of themselves?"

"Always mindful, one breathes in; mindful, one breathes out."

"While breathing in long, one knows: 'I breathe in long.' While breathing out long, one knows 'I breathe out long." ",

"While breathing in short, one knows: 'I breathe in short.' While breathing out short, one knows 'I breathe out short.",

"One trains oneself: Sensitive to the whole body, I breathe in, I breathe out."

"One trains oneself: Sensitive to sensations, perceptions and feelings, I breathe in, I breathe out."

"One trains oneself: Sensitive to mental formations, I breathe in, I breathe out."

"One trains oneself: Sensitive to impermanence and change, I breathe in, I breathe out."

From the Anapanasati Sutra, "Discourse on Full

Awareness of Breathing" (c. 500 BC)

According to this discourse, the breath provides the vehicle, as well as the central gateway, by which mindfulness is developed and insight gained into the nature of those internal and external processes and events that humans are capable of perceiving and understanding. There are a number of methods that are used in different Buddhist schools of insight meditation, but virtually all of them start, at least, with awareness of those physical sensations directly associated with breathing. Initial instructions are to attend to the breath either at the base of the nostrils or the belly without consciously attempting to alter the breathing pattern in any manner. Thus, the initial focus is upon the maintenance of continuous sensory awareness of respiration (e.g., flow and depth, frequency and regularity) breath after breath, over time. As skill and concentration develops, there is a gradual expansion and elaboration that proceeds from the breath itself to other physical sensations more and less directly tied to the physical process of breathing. And from there to increasingly complex mental phenomena, first including basic perceptual aggregates and then proceeding to awareness of emotions, thoughts and other mental events and processes (images, memories, etc.). Ultimately, by means of regular and continuous practice of such inner participant-observation and inquiry, it is thought that one gradually attains a new understanding of the self, the world, and the nature of experience, an understanding inextricably bound to a steady (but continuously changing) state of moment-to-moment awareness. In Buddhist psychology, this is seen not merely as a corrective process but as a transformative one, resulting in an alteration of basic concepts of self, other, society, and natural phenomena.

The stages of actual meditation practice often follow a natural progression based on length and intensity of experience and correspond to the four foundations of mindfulness in the classical formulation (Mahasatipatana sutra; Thera 1962): The body-pleasant, unpleasant, and neutral perceptions; affective states; thoughts and other perceptible mental processes; and the qualities of experience can described as impermanence and disidentification with the usual notion of the self (see Fig. 1 and later discussion).

\section{Mindfulness as Related to an Evolutionary Biological Theory of Consciousness}

The "Discourse on Full Awareness of Breathing" sketches the stages of this transition from sensory awareness to ever more complex mental processes employing the breath as the central thread or anchor of consciousness (e.g., Rosenberg 1998). It may be instructive to contrast this version of learned enhanced awareness with a neurophysiologically based developmental and evolutionary model of consciousness described by two renowned neurobiologists (Edelman and Tononi 2000). In their theory, the earliest dimensions of conscious awareness, both evolutionarily and developmentally, comprise bodily distinctions, primarily mediated via structures in the brainstem that map states of the body in reference to external and internal conditions. These involve proprioceptive, kinesthetic, somatosensory, and other sensory and autonomic components. According 


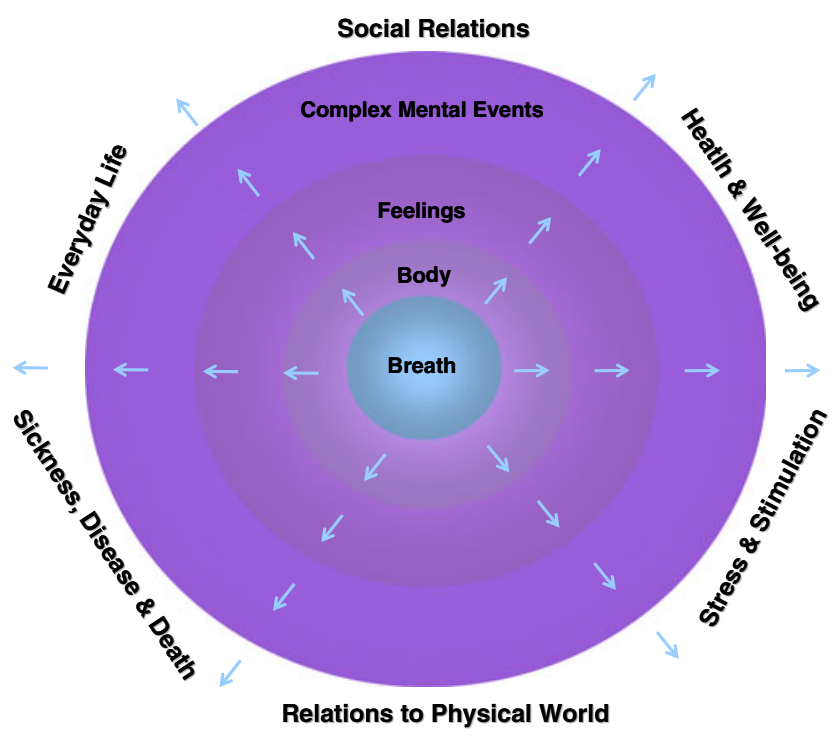

Fig. 1 The systematic structure of mindfulness of breathing. Awareness progressively proceeds from the physical qualities of the breath to other bodily sensations, perceptions, feelings, emotions, and other mental states, purportedly leading to greater insight into inner and outer experience. This developmental model corresponds to the four foundations of mindfulness described in the text.

to the authors, this earliest conscious experience concerns bodily functions we typically remain dimly aware of but which influence every aspect of our being (referred to as the protoself). Later, certain perceptual and emotional dimensions gradually come into play that have become biologically adaptive, having served to promote species survival: For example, increased perceptual discrimination provide a greater flexibility to respond appropriately to important environmental contingencies, and emotional awareness acts as an additional more plastic sentry, supplementing (but in no way supplanting) premammalian stereotypes of approach and avoidance. At the next stage, nonverbal memories have developed to facilitate imagery and provide differentiation of categories of experience: This last stage is seen as characteristic of higher mammals or prelinguistic children. It is only with the development of language and language's ability to manipulate complex symbols that Edelman and Tonino speak of the nascent development of higher human consciousness: "With emergence of higherorder consciousness, true subjectivity emerges with its narrative and metaphorical powers and concepts of self, and of past and future, with interlacing fabric of beliefs and desires that can be expressed. Fiction becomes possible."

This neurobiological theory is, of course, speculative, but does reflect current scientific thinking about the evolution of higher consciousness among humans, an increasingly popular subject among neuroscientists. What may be of particular interest here is the degree to which the systematic development of mindful awareness seems to retrace the evolution of consciousness, starting out as it does with bodily sensations, progressing to ever more complex mental states, and culminating in some new integrative understanding of self and nature. According to Buddhist psychology, lack of nonevaluative moment-to-moment awareness generates "desires and beliefs" central to the self-delusions and fictions we create. In effect, human qualities of complex narrative powers, self-attribution, and formulations of past and future, may often serve to create fictions when they go untempered by a nonevaluative and discerning mind grounded in mindful awareness. On the other hand, according to Buddhist theory, these same capabilities of higher consciousness - when united to dispassionate, discerning, and nonevaluative moment-to-moment awareness - transform to a more accurate understanding and insight into the self and its relationships in the world.

Both the scientific "consciousness" literature and Buddhist psychological treatises, therefore, acknowledge the human capacity for higher conscious awareness. However, a major distinction between these disciplines is that the neuroscience-oriented consciousness literature does not make the distinction between the capacity for conscious experience and the actual performance or act of conscious awareness. Humans are implicitly or explicitly assumed to be conscious, aware creatures at all times, and there is little or no serious discussion of variations in the normal range of conscious awareness nor consideration of the possibility of enhancing conscious awareness. The Buddhist approach to conscious awareness - and one most of us can easily verify by our own personal experience- - is that humans have the capacity to maintain conscious awareness of mental states and outward experience in the present moment; but, this capacity is poorly developed and infrequently utilized, i.e., we are often operating on "automatic pilot", may be all but oblivious to immediate experience and may be absorbed by thoughts or ruminations primarily related to past or future, but unreflective of them. This very lack of awareness, in Buddhism, is seen as the source of the "interlacing fabric of desires, beliefs and fictions" that Edelman and Tononi associate with higher consciousness. According to Buddhist thought, only cultivation of enhanced mindful awareness will result in more veridical perceptions of the self and the world. Consequently, it may only be by exercising awareness from earliest developmental/evolutionary levels onward that this can be achieved, and the systematic development of the four foundations of mindfulness is the method employed. Starting with the breath as an object of mindful awareness may be a particularly skillful approach.

\section{Mindfulness: Starting with the Breath}

The breath, as already mentioned, serves a special function in insight meditation, as well as in many other Eastern 
disciplines that are devoted to promoting alterations of awareness. From a psychophysiological perspective, there are many reasons why respiratory processes provide an ideal point of departure for the practice of developing mindful awareness. First and possibly most obviously, respiration is the one continuous and vital physiological function that is accessible to sensation and perception in all situations and at all times, until finally extinguished by death. The act of breathing is readily available to each our senses - touch, taste, olfactory, auditory, visual, proprioceptive, kinesthetic, and interoceptive. Also, because the breath is so clearly essential to life itself and yet so typically unexamined by the breather, it is an inherently compelling object of attention-always there, always sustaining life, rarely looked at, but with a potential for flooding consciousness with swells of sensory information.

The lungs are the largest set of organs in our body and act as continuous pumps during the respiratory phases of inhalation and exhalation. This means that the breathing system also is the most powerful physiological oscillator in our chests and our bodies. True to the physical properties of strong oscillators, the rhythm of the lungs entrains other weaker rhythmically oscillating physiological systems, like the heart beat, blood pressure, and central nervous system activity (Grossman 1983). Attending to the breath, therefore, implies observing a powerful, physically resonating force in the body, which directly or indirectly influences virtually all other vital processes.

Not only the sensory flood and oscillating force of breathing make it a valuable initial object of attention, but the pattern of respiration and the rate at which we breathe conform well to those perceptual capabilities of human attention: The breathing pattern is not constant from breath to breath, but changes either subtly or markedly, dependent upon internal and external conditions and thus satisfies a natural human tendency to orient to novel stimuli. Additionally, humans are capable of processing sensory stimuli at a rate of at least $20-30 \mathrm{~Hz}$ (i.e., 20-30 stimuli per second). With respect to the breathing cycle, all significant information is captured within the range of this perceptual capability. In fact, physiological studies of respiration rarely digitally sample or record the respiratory waveform at frequencies beyond $30 \mathrm{~Hz}$ because this frequency already provides enough data points to trace all significant dimensions of the breathing pattern for scientific inquiry. Contrast this with the electrocardiogram, which records the electrical activity of the heart pump. The cardiac cycle generally occurs at a frequency at least four times faster than the breathing pattern (e.g., about 70 beats per min vs. approximately 15 breaths per $\mathrm{min}$ ). In addition, the electrocardiogram is a complex of a number of different waveforms occurring during each heartbeat, in contrast to the single, essentially sinusoidal waveform that character- izes each breath. To accurately depict all cardiac events, one needs to have information every millisecond $(1,000 \mathrm{~Hz}$ vs. $30 \mathrm{~Hz}$ ). Even if we could directly sense our cardiac events - which we typically cannot - we would still miss out on much relevant information.

Mindful awareness of breathing has still several other dimensions that are relevant here but may not be obvious. The respiratory system is unique because it can function almost completely under conscious control or completely under unconscious control (e.g., Phillipson et al. 1978). A comatose patient with only the most primordial part of the brain remaining intact, i.e., the brainstem, will often be able to breathe with no mechanical assistance and therefore achieve the major aim of respiration, i.e., the exchange of vital gases $\left(\mathrm{O}_{2}\right.$ and $\left.\mathrm{CO}_{2}\right)$ between lungs and atmosphere. On the other hand, awake humans are able to exert enormous conscious, voluntary control over breathing. In fact, specific cerebral and midbrain respiratory areas have been identified that come into play during conscious alteration of breathing pattern. Indeed, as children, we must learn to adapt our ventilatory pattern to various behavioral activities during daily life (e.g., speaking, eating, and singing), and experimental learning studies indicate the ease of conditioning parameters of breathing (Van den Bergh et al. 1997). Thus, respiration lies precisely at the juncture of conscious and unconscious experience, voluntary and involuntary behavior. Developing mindfulness of the breath should expose to awareness this meeting point between controllable and uncontrollable, conscious and unconscious physiological processes, as well as give us insight into the habitual patterns of respiration learned over the course of our lives, which may, perhaps, constitute elemental examples of functioning on "automatic pilot." Mindful awareness of breathing may also provide us with information about any tendencies we may have to exert conscious control over a physiological process that, under many circumstances, ordinarily can take care of itself.

The "Discourse on Full Awareness of Breathing" additionally enlists the breath in order to gain awareness of processes well beyond the physical sensations of respiration. Here too, the breath is well-suited to this purpose, since the respiration pattern is exquisitely sensitive to emotional states and behavioral activities (Boiten 1998; Grossman and Wientjes 2001). Emotions such as fear, anger, anxiety, and happiness are related to specific breathing patterns, as are variations in mental processes, such as problem solving or mental calculation. Breathing pattern is even sensitive to different stages of sleep (Guilleminault et al. 2001). By maintaining moment-to-moment awareness of the breath over time and across fluctuating emotional states, one begins to discern relationships between physiology and mental processes and can employ the breath as a vehicle to examine emotions and other mental states. 
In a very concrete sense, bringing the breath into moment-to-moment awareness enables us, like that of no other process, to gain practical and personal insight into how mind and body interact under varying conditions of repose and activity. Respiration is the only physiological system with continuous perceptible output that can be related to mental states and brought into conscious, immediate experience. In the current "scientific" era, we often rely upon nomothetic, normative data to understand even our own inner workings. Precisely because of its normative quality, such information is typically flawed when it comes to within-subject relationships and may consequently bias how we interpret own patterns of individual functioning in the direction of the statistically "average" response. Our own characteristic responses and psychophysiological relationships are likely to vary from such mean tendencies. As such, mindful awareness of breathing offers an introspective method to explore personal psychophysiological relations, a method of inquiry that supplants the nomothetic, normative approach we usually take to comprehend our own mind-body relations.

Finally, in regard to employing the breath in mindfulness, the very act of attending to breathing slows and deepens respiration-even in the absence of conscious effort to manipulate the pattern (Western and Patrick 1988). This slower, deeper ventilation has been found to elicit a greater sense of calm which, in turn, is likely to promote a more concentrated and nonevaluative awareness of the present moment (see Grossman 1983).

All these aspects of the breath-its accessibility, its physiological uniqueness in terms of control mechanism and its intimate relationship to higher mental processes-provide a unique object of awareness that is optimally suited for developing understanding and insight into how mind and body are intricately bound together. Although many methods of insight meditation only focus upon the breath during a preliminary phase in order to develop concentration and the ability to sustain attention to a single object of awareness, mindfulness of breathing can be a method of practice complete within itself (Rosenberg 1998). Furthermore, awareness of breath plays a major and continuous function in the MBSR program (Kabat-Zinn 1990).

\section{The Buddhist Mindfulness Approach vs. Western Behavioral Sciences: Some Salient Distinctions}

Three decades ago, Walsh (1980) characterized essential differences between the prevailing behavioral science paradigm and what he termed the "consciousness discipline" paradigm, an approach that addresses systematic alterations in states of consciousness. In this classic article, Walsh made several distinctions that are still highly relevant today and may be of particular value for therapists and researchers contemplating application of some aspect of mindfulness as a clinical intervention. Mindfulness practice fits well in Walsh's conceptualization of consciousness disciplines and warrants a discussion below, where I integrate his ideas with several of my own. The notion of a consciousness discipline will be further clarified by the explanations and examples below.

\section{Practical Experience vs. Intellectual Knowledge}

That the practice of mindfulness is radically different than Western psychological and behavioral approaches may already be inferred from the previous discussion. Nevertheless, it is useful to make such distinctions as explicit as possible, particularly for those clinicians and academics who are interested in the application of mindfulness practice in client and patient populations.

Perhaps the most critical differences between approaches from a professional and clinical standpoint have to do with the role of practical experience vs. intellectual knowledge. The Buddhist approach to mindfulness is founded upon an intensive and continuous, personal practice of mindfulness meditation. Unique knowledge is thought to derive from such individual exploration and inquiry. This kind of knowledge is seen as inherently different from and not interchangeable with the intellectual or theoretical learning of a technique. The most direct implication of this premise for therapists interested in mindfulness as a clinical intervention is that substantial and prolonged selfexperience with mindfulness is considered a prerequisite for helping others to develop mindful awareness (Kabat-Zinn 2003; Segal et al. 2002).

Intellectual endeavors are not depreciated in this view but represent a separate related source of knowledge. Nevertheless, the acquisition of mindfulness is seen to have important transformative properties that transcend and cannot be completely understood by intellectual analysis (Teasdale et al. 2002). For example, the very concept of the "self," is thought to become profoundly altered as a consequence of mindfulness practice. This type of change cannot be comprehended outside of one's own experience, making it difficult, if not impossible, to teach mindfulness without practicing it oneself. Hence, the "insight" of insight meditation derives from the participant-observational awareness of one's own mental states and events. The idea is that it cannot be acquired by any other means, such as the mere study the techniques involved nor analysis of the putative effects that mindful aware may produce. Support of the process among participants in a MBSR program, for example, requires symmetrical engagement in mindfulness practice of participants and the course leader.

Such emphasis upon extended introspective experience is at significant variance with most Western psychotherapeutic 
approaches that place limited or sometimes no value upon the self-experience of therapists with regard to those psychotherapeutic methods and interventions they employ (a notable exception being psychoanalysis). Training in therapy normally implies intense analysis of psychotherapeutic skills and techniques in relation to psychological and behavioral benefits, rather than shared personal experiences related to the prolonged application of the methods to the therapists themselves. Most psychotherapeutic interventions, furthermore, are aimed at modifying specific behaviors or cognitions in a previously operationalized manner and direction - not at transformation of attitudes, mindsets, and experience into a radically new structures that may take multiple, open-ended forms.

\section{The Nature of Investigated Phenomena}

In Western behavioral approaches, the fundamental focus is often upon quantifiable, verifiable, and statistically valid entities related to behavioral and psychological functioning, i.e., measurable behavioral responses, self-report scales, or structured clinical interview evaluations. Such data are used to characterize and classify normal and dysfunctional psychological states and traits, often with the objective of therapists treating the behavioral disorders or at least modifying the behavior of another person.

Proponents of Buddhist mindfulness and other consciousness disciplines, on the other hand, make claims that normal states of functioning, themselves, are suboptimal; they generally do not distinguish the normal from the psychopathological. In Buddhist psychology, the normal everyday state of consciousness is seen as an often deluded one, in which the individual is operating in a state below full wakefulness. Accordingly, Buddhist psychology maintains that it is possible to achieve states of consciousness and insight into the nature of the mind and reality that are far beyond those considered by most Western psychologies. Additionally, mindfulness is an approach toward understanding conscious experience that is without any preconceived classification. It is not primarily interested in quantification, categorization, understanding, or modifying the psychological functioning of others, but rather in the development, within oneself, of those states of consciousness associated with greater awareness and insight into the workings of the mind and all natural processes amenable to human perception and understanding. Consequently, the basic currency of mindfulness is consciousness and its many variations, or states. Classification systems that arise in Buddhist psychology are those that attempt to clarify such mental states and processes and are very elaborate, indeed (Goleman 1988), when compared with the relatively recent mentalistic concepts of cognitive-behavioral psychology.
In contrast, states of consciousness tend not to be seriously considered in Western behavioral traditions, with few exceptions (e.g., James 1958). In fact, the very concept of "state of consciousness" seems all but missing in formal mainstream Western psychologies. A search of the US National Library of Medicine and National Institute of Health PUBMED website found not a single hit for the phrase "states of consciousness." Psychologists do talk about "states" and "traits", but these are usually delimited to specific affective dimensions (i.e., state vs. trait anxiety, anger, and so on), rather than capturing a broad range of simultaneously occurring emotional, cognitive, social, and physiological aspects acting together to produce and depict a particular state of consciousness. This may be the consequence of a scientific orientation toward discrete and circumscribed psychological categories, a kind of behavioral reductionism.

Although sleep states, drug states, and pathological states of consciousness are sometimes acknowledged, the only other implicit option appears to be the normal waking state. Indeed, there are numerous states of consciousness during sleep that are operationalized by EEG and other physiological and behavioral measures during polysomnography studies, but classification ceases when the person being measured wakes up (here, there is a single category of being "awake"'). This, however, is just where Buddhist psychology starts. Western psychophysiologists may occasionally examine variations in psychological and physiological functioning under different physiological conditions (even very subtle ones like posture change from lying to sitting; Sloan et al. 1995) but do not conceptualize such differences in terms of detectable variations in state of consciousness, even though assorted and correlated physiological, psychological, behavioral, and social activities do markedly vary-even from posture to posture. It is just these kinds of more and less subtle changes that are the very stuff of mindfulness, the continual and often non-discrete flux of human consciousness in its many variegated, perceptible forms. Often, Western psychology treats the concept of "states of consciousness" and approaches such as mindfulness largely with skepticism and disdain: If there is no clear notion of states of consciousness engrained in behavioral science traditions and no tradition of training of conscious awareness, then the ideal of higher states of consciousness and their development — so essential to Buddhist psychology—may appear to some as ludicrous and without basis. For consciousness disciplines, on the other hand, the personal experience of varied states of consciousness make their validity self-evident.

Personality, Ego, and the Self

The concepts of a stable ego, personality, or self are pervasive to Western psychological theory and applications. 
The notion of a relatively enduring and coherent set of psychological traits characteristic of the individual is, in fact, one of the very foundations of Western psychology, although the concept of personality stability has been challenged and modified in the psychological literature over the years (Mischel 2004; Roberts and DelVecchio 2000). It may be just this central role of stable individual dispositions in behavioral sciences that makes state of consciousness an untenable or, at least, uncomfortable idea: If states of consciousness are transformative, behavioral dispositions are then unstable and subject to change as consciousness is altered. This obviously challenges the idea that the personality is fixed. In fact, recent research has indicated that even short-term test-retest correlations of personality tests - one very important measure of stability rarely indicate very high levels of stability, with most coefficients typically around 0.55 or lower (accounting for $30 \%$ of the personality trait variance or less as stable over even a year or two; Roberts and DelVecchio 2000). Stability coefficients across many years are even much lower. Thus, there is only limited direct evidence of the constancy of personality structure. Furthermore, a host of often-changing factors seems to influence consistency of measures of personality or self concept. Besides genetic influences (which, incidentally, can also vary across time), they include environmental factors, person-environment transactions, and genetic-person-environment interactions.

Buddhist psychology, on the other hand, considers the idea of a well-delineated, largely invariant self, or individual sense of identity, as an illusion conditioned by a mind not trained in sustaining nonjudgmental moment-to- moment mindful awareness. In Buddhist psychology, the conventional concept of self is seen as little more than a loosely held-together aggregate of transient elements of mind and body that include the body itself, sensations, perceptions, thoughts, and any other mental states impinging upon consciousness (Goldstein 1993; Rosenberg 1998). According to Buddhist ideas, we cling to an illusory sense of consistent identity fueled by desires and aversions and resulting from our inability to maintain mindful awareness. Paying attention to experience in the present moment makes clear just how impermanent and changing all those qualities are that we consider our "selves": Our bodies change in appearance and actual physical makeup over time, and our sensations, perceptions and thoughts are in constant flux. Therefore, within this conception, a firm sense of self does, indeed, appear difficult to justify. According to Buddhist thought, only through the systematic and continued practice of mindful awareness does it become clear that actual experience is replete with all sorts of changing mental states and physical sensations and that our usual concept of self is inaccurately restrictive both in defining intrinsic qualities and fixing them across time and space. A conventional sense of self clearly remains, but we begin to see how fluid, permeable, and ephemeral many qualities of "self" really are.

In sum, Buddhist psychology focuses on the processes and transformation of conscious awareness and regards constancy of personality or self for the most part as an empty fiction. Western psychologies, on the other hand, emphasize the importance of enduring psychological traits in both practical and theoretical matters (e.g., psychother-

Table 1 A contrast of central concepts of the mainstream behavioral science paradigm with the conscious disciples, including insight meditation and mindfulness practice

\begin{tabular}{ll}
\hline Behavioral Science Paradigm & Consciousness Disciplines \\
\hline Verifiable phenomena central & Consciousness is central concern \\
Normal, waking consciousness optimal & Usual consciousness extremely suboptimal \\
Variety of states of consciousness ignored or pathologized & Multiple states of consciousness exist \\
Skepticism or denial of higher states of consciousness & Higher states of consciousness achievable \\
No tradition of consciousness training & Extensive mental training necessary \\
Goal: ME happy (some traditions of self-actualization) & Ultimate goal of liberation, awakening \\
Happiness=stimulation, novelty, activity, accumulation, and power & Happiness=peace, equanimity, compassion; freedom from attachment \\
& and accumulation \\
Psychotherapy and healthy ego stand central & Psychotherapeutic aspects largely neglected \\
Ultimate assessment must be scientific, intellectual, and behavioral & Approach inherently introspective \\
Optimal and only path to knowledge thru intellect & Language, abstract thought insufficient for understanding \\
Exclusively based on self-reports or observation of others & Completely grounded in personal experience \\
Self-report and observational measures often naïve, limited & Range and depth of personal experience \\
Clear ego boundaries, psychological stability, and individuality & Buddhism: fundamental concepts of no-self, impermanence \\
Corrective: modify avoidant, evaluative and dysfunctional behavior & Constructivist: expands range and flexibility of capacities \\
Clear separation of cognitive, emotional, and moral realms & Fluid integration of cognitive, emotional and moral realms \\
\hline
\end{tabular}


apy and personality theory) and tend to ignore "states of consciousness" as explanatory principles that might, at least partially, account for the vagaries of mental traits. Obviously, these conflicting viewpoints must be considered when mindfulness is applied as a clinical intervention by therapists primarily schooled in the behavioral science paradigm.

Most of Western psychological theory and therapeutic application is consequently aimed at the strengthening of the ego, enhancement of self-esteem, or "getting one's needs met." The goals are often specific (successful treatment of depression, anxiety, borderline syndrome, trauma, etc.) and inherently individual in nature-my problem, my dysfunction, my unhappiness. In Buddhist psychology, the individual dimension falls away to a significant extent, and the aims of meditation practice are seen as more universal and not primarily psychotherapeutic, in nature. These qualities are usually framed in terms of awakening, more accurate perception, fuller awareness of all experience, and liberation from the bonds of desires and aversions. Buddhist psychology makes no distinctions between depressives, anxiety neurotics and "normal" individuals but suggests that the process of development of awareness is universally available and generally follows a common, if temporally varying, path. In effect, insight, itself, is the goal, rather than the fixing a problem or attainment of personal happiness, as the phrase is typically understood.

\section{Conclusions and Limitations}

A couple of notes of warning, however, are necessary. Due to the brevity of discussion, I certainly have painted an oversimplistic picture of differences between the behavioral science paradigm and the Buddhist one. Surely, there are similarities as well as differences, particularly regarding some developed in Western behavioral science, e.g., transpersonal and existential psychology. Nevertheless, some appreciation of differences between approaches may aid psychologists and therapists when thinking about mindfulness as a clinical intervention. An overview of several of these distinctions is provided in Table 1. Also, Buddhist psychology is certainly not monolithic, and I may have interpreted certain Buddhist concepts in ways that are open to debate.

Nevertheless, the foregoing discussion of mindfulness has hopefully clarified some of the implicit and explicit assumptions of MBSR and other mindfulness-based interventions, so that empirical evaluation of the relevant literature can be more meaningfully examined. When interpreting the scientific findings, it may be particularly important to keep in mind that MBSR and mindfulness practice are not directed toward curing the individual of their presenting complaints (although this may sometimes occur) or inducing some kind of relaxation response that can generalize to everyday activities. In fact, little or no emphasis is placed on treating participants for their symptoms or promising them direct relief. Mindful awareness, as previously explained, is oriented toward an improved understanding of one's perceptible and comprehensible inner and outer world. Any measurable benefits of mindful awareness must be considered from this background. Therefore, the usual psychosocial, dependent variables employed to assess effects of intervention are probably best understood within the context of some more fundamental psychological—or even existential—processes than mere symptom or stress reduction.

In conclusion, it is my hope that greater awareness of the issues raised in this paper, and elsewhere, may serve to safeguard the concept of mindfulness from becoming denatured, banalized, distorted, or otherwise reified. In turn, this may enhance the likelihood of mindfulness-based approaches to achieve fuller potential in their applications to critical aspects of wellbeing, quality of life, and healthcare.

Acknowledgments This investigation was supported by grants from the Swiss National Science Foundation, The Swiss Multiple Sclerosis Society, The Swiss Cancer League, and the Samueli Institute (Corona del Mar, CA, USA).

\section{References}

(1995). Roget's II: The New Thesaurus, 3rd edn. New York: Houghton Mifflin Company.

Boiten, F. A. (1998). The effects of emotional behavior on components of the respiratory cycle. Biological Psychology, 49, 29-51.

Buchheld, N., Grossman, P., \& Walach, H. (2001). Measuring mindfulness in insight meditation (Vipassana) and meditationbased psychotherapy: The development of the Freiburg Mindfulness Inventory. Journal of Meditation Research, 1, 11-34.

Depraz, N., Varela, F., \& Vermersch, P. (2003). On becoming aware. Amsterdam: John Benjamins.

Edelman, G., \& Tononi, G. (2000). A universe of consciousness. NY: Basic Books.

Epstein, M. (1995). Thoughts without a thinker: Psychotherapy from a Buddhist perspective. NY: Basic Books.

Goldstein, J. (1993). Insight meditation. Boston: Shambhala.

Goleman, D. (1988). The meditative mind: The varieties of meditative experience. New York: Dutton.

Grossman, P. (1983). Respiration, stress, and cardiovascular function. Psychophysiology, 20(3), 284-300.

Grossman, P., Niemann, L., Schmidt, S., \& Walach, H. (2004). Healthrelated benefits of mindfulness-based stress reduction: A metaanalysis. Journal of Psychosomatic Research, 57, 35-43.

Grossman, P., \& Wientjes, C. J. (2001). How breathing adjusts to mental and physical demands. In Y. Haruki, I. Homma, A. Umezawa, \& Y. Masaoka (Eds.), Respiration and emotion (pp. 43-55). New York: Springer. 
Guilleminault, C., Poyares, D., Palombini, L., Koester, U., Pelin, Z., \& Black, J. (2001). Variability of respiratory effort in relation to sleep stages in normal controls and upper airway resistance syndrome patients. Sleep Medicine, 2, 397-405.

James, W. (1958). The varieties of religious experience. NY: New American.

Kabat-Zinn, J. (1990). Full catastrophe living: Using the wisdom of your body and mind to face stress, pain and illness. NY: Delacorte.

Kabat-Zinn, J. (1993). Mindfulness meditation: health benefits of an ancient Buddhist practice. In D. Goleman \& J. Gurin (Eds.), Mind/body medicine (pp. 259-275). NY: Consumer Report Books.

Kabat-Zinn, J. (2003). Mindfulness-based interventions in context: Past, present, and future. Clinical Psychology: Science \& Practice, 10, 144-156.

Langer, E. (1997). Power of mindful learning. Reading: AddisonWesley.

Mischel, W. (2004). Toward an integrative science of the person. Annual Review of Psychology, 55, 1-22.

Nanamoli, B., \& Bodhi, B. T. (1995). The middle length discourses of the Buddha. Boston: Wisdom Press.

Phillipson, E., McClean, P. A., Sullivan, C. E., \& Zamel, N. C. (1978). Interaction between metabolic and behavioral repsiratory xontrol during hypercapnia and speech. The American Review of Respiratory Disease, 117, 903-909.

Roberts, B. W., \& DelVecchio, W. F. (2000). The rank-order consistency of personality traits from childhood to old age: A quantitative review of longitudinal studies. Psychological Bulletin, 126, 3-25.

Rosenberg, L. (1998). Breath by breath: The liberating practice of insight meditation. Boston: Shambhala.
Segal, Z. V., Williams, J. M. G., \& Teasdale, J. D. (2002). Mindfulness-based cognitive therapy for depression: A new approach to relapse prevention. NY: Guilford Press.

Shapiro, S. L., \& Schwartz, G. E. (2000). Intentional systemic mindfulness: an integrative model for self-regulation and health. Advances in Mind-Body Medicine, 16, 128-134.

Sloan, R. P., Shapiro, P. A., Bagiella, E., Fishkin, P. E., Gorman, J. M., \& Myers, M. M. (1995). Consistency of heart rate and sympathovagal reactivity across different autonomic contexts. Psychophysiology, 32, 452-459.

Sternberg, R. J. (2000). Images of mindfulness. Journal of Social Issues, 56, 112-126.

Teasdale, J. D., Moore, R. G., Hayhurst, H., Pope, M., Williams, S., \& Segal, Z. V. (2002). Meta-cognitive awareness and prevention of relapse in depression: empirical evidence. Journal of Consulting \& Clinical Psychology, 70, 278-287.

Thera, N. (1962). The heart of Buddhist meditation. New York: Weiser. Van den Bergh, O., Stegen, K., \& Van de Woestijne, K. P. (1997). Learning to have psychosomatic complaints: Conditioning of respiratory behavior and somatic complaints in psychosomatic patients. Psychosomatic Medicine, 59, 13-23.

Varela, F., Thompson, E., \& Rosch, E. (1991). The embodied mind: Cognitive science and human experience. Cambridge: MIT Press.

Walsh, R. (1980). The consciousness disciplines and the behavioral Sciences: Questions of comparison and assessment. The American Journal of Psychiatry, 137, 663-673.

Webster's Collegiate Dictionary. (1998) Tenth Edition New York: Simon and Schuster.

Western, P. J., \& Patrick, J. M. (1988). Effect of focusing attention on breathing with and without apparatus on the face. Respiration Physiology, 72, 125-130. 